\title{
A risk factor-based predictive model for new-onset hypertension during pregnancy in Chinese Han women
}

Yamin $\mathrm{Hou}^{1,2+}$, Lin Yun ${ }^{3+}$, Lihua Zhang ${ }^{3}$, Jingru Lin ${ }^{4}$ and Rui $\mathrm{Xu}^{1,2^{*}}$

\begin{abstract}
Background: Hypertensive disorders of pregnancy (HDP) is one of the leading causes of maternal and neonatal mortality, increasing the long-term incidence of cardiovascular diseases. Preeclampsia and gestational hypertension are the major components of HDP. The aim of our study is to establish a prediction model for pregnant women with new-onset hypertension during pregnancy (increased blood pressure after gestational age $>20$ weeks), thus to guide the clinical prediction and treatment of de novo hypertension.
\end{abstract}

Methods: A total of 117 pregnant women with de novo hypertension who were admitted to our hospital's obstetrics department were selected as the case group and 199 healthy pregnant women were selected as the control group from January 2017 to June 2018. Maternal clinical parameters such as age, family history and the biomarkers such as homocysteine, cystatin $\mathrm{C}$, uric acid, total bile acid and glomerular filtration rate were collected at a mean gestational age in 16 to 20 weeks. The prediction model was established by logistic regression.

Results: Eleven indicators have statistically significant difference between two groups $(P<0.05)$. These 11 factors were substituted into the logistic regression equation and 7 independent predictors were obtained. The equation expressed including 7 factors. The calculated area under the curve was $0.884(95 \%$ confidence interval: 0.848-0.921), the sensitivity and specificity were 88.0 and $75.0 \%$. A scoring system was established to classify pregnant women with scores $\leq 15.5$ as low-risk pregnancy group and those with scores $>15.5$ as high-risk pregnancy group.

Conclusions: Our regression equation provides a feasible and reliable means of predicting de novo hypertension after pregnancy. Risk stratification of new-onset hypertension was performed to early treatment interventions in high-risk populations.

Keywords: Hypertension, pregnancy induced, Prediction model, Risk factors, Homocysteine

\footnotetext{
* Correspondence: xuruicn@hotmail.com

${ }^{+}$Yamin Hou and Lin Yun contributed equally to this work.

'Department of Cardiology, Shandong Provincial Qianfoshan Hospital,

Shandong University, Jinan 250014, P.R. China

${ }^{2}$ Department of Cardiology, The First Affiliated Hospital of Shandong First

Medical University, Jinan 250014, P.R. China

Full list of author information is available at the end of the article
}

(C) The Author(s). 2020 Open Access This article is licensed under a Creative Commons Attribution 4.0 International License, which permits use, sharing, adaptation, distribution and reproduction in any medium or format, as long as you give appropriate credit to the original author(s) and the source, provide a link to the Creative Commons licence, and indicate if changes were made. The images or other third party material in this article are included in the article's Creative Commons licence, unless indicated otherwise in a credit line to the material. If material is not included in the article's Creative Commons licence and your intended use is not permitted by statutory regulation or exceeds the permitted use, you will need to obtain permission directly from the copyright holder. To view a copy of this licence, visit http://creativecommons.org/licenses/by/4.0/ The Creative Commons Public Domain Dedication waiver (http://creativecommons.org/publicdomain/zero/1.0/) applies to the data made available in this article, unless otherwise stated in a credit line to the data. 


\section{Background}

Hypertensive disorders of pregnancy (HDP) is a common placental-mediated syndrome complicated by various types of diseases during pregnancy and is also one of the leading causes of maternal and neonatal mortality. The disease manifests as hypertension, proteinuria and edema and is associated with serious complications such as hemolysis, elevated liver enzymes, and low platelet count (HELLP) syndrome, placental abruption, and stillbirth. Despite extensive clinical and basic research on HDP in recent decades, the real etiology and pathophysiology remain unclear $[1,2]$. According to the latest recommendations from the International Society for the Study of Hypertension in Pregnancy (ISSHP), HDP includes pregnancy complicated by chronic hypertension (high blood pressure before pregnancy or increased blood pressure before a gestational age of 20 weeks) and new-onset hypertension [gestational hypertension $(\mathrm{GH})$ or preeclampsia (PE)] [3]. PE and $\mathrm{GH}$ are the major components of HDP; their worldwide incidences are approximately $1.8-4.4 \%$ and $0.2-9.2 \%$, respectively, and their incidences have regional and seasonal differences [4-6]. HDP increases the long-term incidence of cardiovascular diseases in pregnant women. Many studies have shown that the incidence of cardiovascular diseases in HDP patients is 2 times higher than that in normal pregnant women [7]. The occurrence of chronic hypertension in HDP patients is 1.5 times higher than that in normal pregnant women [8]. PE is an independent risk factor for the subsequent hypertension [9]. Basal blood pressure and the long-term incidence of cardiovascular diseases are higher in children born by pregnant women with hypertension [10]. The personal and social burden caused by HDP is very serious. Therefore, studies on the risk factors of HDP have always been on-going.

Currently, the most studied risk factors include maternal age, multiple pregnancies, obesity, and family history of $\mathrm{PE}$, previous $\mathrm{PE}$, chronic hypertension, and genetics. However, the specificity and sensitivity of these risk factors remain questionable [11-14]. There are many risk factors for HDP, and none of them have been used as a gold standard for HDP screening. A study demonstrated that none of the classical risk factors alone reached an acceptable discriminatory accuracy [15]. The establishments of risk factor models have mostly focused on predicting the risk of PE and have not concentrated on $\mathrm{GH}$. However, the ISSHP believes that the incidence of GH is constantly increasing and that $\mathrm{GH}$ will convert to PE in some patients in the late stage, and it classifies HDP as chronic hypertension and de novo hypertension during pregnancy ( $\mathrm{GH}$ and $\mathrm{PE}$ ). The ISSHP also questions the existing diagnostic criteria for PE, suggesting that proteinuria cannot be used as a mandatory criterion for the diagnosis of $\mathrm{PE}$ and that if liver and kidney dysfunction are present, PE needs to be considered. Therefore, the purpose of this study was to combine the clinical and biochemical indices of patients to establish a risk model for new-onset hypertension ( $\mathrm{GH}$ and $\mathrm{PE}$ ) in Chinese Han women and to provide a theoretical basis for the prediction and prevention of HDP progression.

\section{Methods \\ Enrolled populations}

Participants who underwent physical examination and gave birth in hospital were recruited from January 2017 to June 2018. Case control study design was adopted. Patients with hypertension during pregnancy were selected as case group (gestational hypertension, preeclampsia) and normal pregnancy as control group.

\section{Case group and control group inclusive and exclusive criteria}

The case group inclusion criteria were as follows: age over 18 , women who had a singleton pregnancy, received antenatal care after 16 to 20 weeks and delivered in the same maternity unit were eligible for inclusion, and diagnosed in gestational hypertension or preeclampsia after discharge and the control group diagnosed no hypertension. The exclusion criteria were as follows: (1) secondary hypertension (primary aldosteronism, pheochromocytoma, Cushing syndrome, etc.), (2) patients with chronic hypertension combined with pregnancy and superimposed preeclampsia, (3) severe heart failure and liver and kidney failure, (4) chronic kidney disease and renal vascular disease, (5) acute and severe diseases in obstetrics and gynecology (amniotic fluid embolism, eclampsia, HELLP syndrome, etc.), (6) tumor, (7) new serious infections, (8) severe anemia (hemoglobin $<60 \mathrm{~g} / \mathrm{L}$ ), (9) multiple organ failure.

\section{Data collection}

Maternal clinical parameters were included age, height, weight, family history of HDP. Maternal fasting blood samples were collected after an overnight fast of at least $8 \mathrm{~h}$ at a mean gestational age of 16 to 20 weeks. The biomarkers were included white blood cell count (WBC), red blood cell count $(\mathrm{RBC})$, hemoglobin $(\mathrm{HB})$ and platelets (PLT), albumin (ALB), globulin (GLB), glucose (Glu), free fatty acids (FFA), direct bilirubin (DBil), indirect bilirubin (IBil), homocysteine (HCY), cystatin C (CYC), uric acid (UA), total bile acid (TBA), serum calcium $(\mathrm{Ca})$, serum zinc $(\mathrm{Zn})$, estimated glomerular filtration rate (eGFR).

\section{Data analysis}

A total of 316 patients were enrolled: 199 healthy pregnant women and 117 pregnant women with GH or PE. The body mass index (BMI) of each sample was 
calculated using patient height and weight. All data were analysed using SPSS23.0 (SPSS Inc., Chicago, IL, USA). Measurement data are expressed as the means \pm standard deviations $(x \pm s)$, except for the HCY, TBA, Dbil, IBil, FFA ( $\left.\mathrm{M}, \mathrm{P}_{25}-\mathrm{P}_{75}\right)$. Counting data are expressed as proportions (\%). An independent-samples t-test was performed for age, CYC, ALB, GLB, Glu, UA, Ca, Zn, BMI, eGFR, WBC, RBC, Hb, and PLT, and Mann-Whitney U tests was performed for HCY, TBA, DBil, IBil, FFA. Meaningful factors were converted to ordinal variables based on percentiles, except the age for five groups cause the age range is small. The obtained ordinal variables and family history were subjected to the Chisquare test to determine the $P$ value. Meaningful factors were subjected to collinearity diagnosis to clarify the presence of collinear interactions among them. After excluding the influence of collinearity, each factor was substituted into a binary logistic regression equation to screen out meaningful independent risk factors and establish a risk factor-based prediction model for pregnancy induced hypertension. The area under the ROC curve (AUC), the optimal cutoff point and the $95 \%$ confidence interval $(\mathrm{CI})$ of the model were calculated to evaluate its validity. The scores were calculated according to the regression coefficient $\beta$ of each variable in the logistic regression prediction model, i.e., the score of each variable was the integer value of the absolute value of each variable $\beta$ divided by the minimum value of the absolute value of each variable $\beta$. The scores for each study subject were calculated to establish a scoring system. The AUC was used for evaluation.

\section{Results}

The difference between the two groups was statistically significant in age $(29.410 \pm 4.803,31.460 \pm 5.253, \quad P<$ 0.001), HCY[10.600(6.600-14.800), 14.000(10.100, 18.900), $P<0.001)], C Y C(1.081 \pm 0.289,1.376 \pm 0.339, P<$ $0.001)$, UA $\quad(275.820 \pm 72.209, \quad 350.040 \pm 94.962, \quad P<$ 0.001), TBA[2.100(1.400-3.200), 3.400(1.500-5.100), $P<$ $0.001]$, BMI $(28.457 \pm 3.348, \quad 30.766 \pm 3.793, \quad P<0.001)$, calcium $(2.148 \pm 0.103,2.081 \pm 0.223, P=0.003)$, zinc $(8.248 \pm 1.599,7.727 \pm 1.972, P=0.016)$, ALB $(33.360 \pm$ 4.136, $29.997 \pm 3.911, P<0.001)$, eGFR $(222.933 \pm 66.143$, $187.506 \pm 58.359, P<0.001)$. However, there was no statistically significant group difference for GLB, FFA, DBil, IBil, Glu, WBC, RBC, PLT and Hb as shown in Table 1.

Table 1 Comparison of basic and clinical indicators between the 2 groups

\begin{tabular}{|c|c|c|c|}
\hline Item & Control group $(\boldsymbol{n}=199)$ & $\begin{array}{l}\text { Case group } \\
(\boldsymbol{n}=117)\end{array}$ & $\boldsymbol{P}$ value \\
\hline Age (yr) & $29.410 \pm 4.803$ & $31.460 \pm 5.253$ & $<0.001$ \\
\hline $\mathrm{HCY}(\mu \mathrm{mol} / \mathrm{L})$ & $10.600(6.600-14.800)$ & $14.000(10.100,18.900)$ & $<0.001$ \\
\hline Height (cm) & $161.580 \pm 4.855$ & $161.210 \pm 4.434$ & 0.497 \\
\hline Weight (kg) & $74.299 \pm 9.257$ & $79.914 \pm 10.076$ & $<0.001$ \\
\hline $\mathrm{UA}(\mu \mathrm{mol} / \mathrm{L})$ & $275.820 \pm 72.209$ & $350.040 \pm 94.962$ & $<0.001$ \\
\hline CYC (mg/L) & $1.081 \pm 0.289$ & $1.376 \pm 0.339$ & $<0.001$ \\
\hline TBA $(\mu \mathrm{mol} / \mathrm{L})$ & $2.100(1.400-3.200)$ & $3.400(1.500-5.100)$ & $<0.001$ \\
\hline Calcium (mmol/L) & $2.148 \pm 0.103$ & $2.081 \pm 0.223$ & 0.003 \\
\hline BMI $\left(\mathrm{kg} / \mathrm{m}^{2}\right)$ & $28.457 \pm 3.348$ & $30.766 \pm 3.793$ & $<0.001$ \\
\hline Zinc $(\mu \mathrm{mol} / \mathrm{L})$ & $8.248 \pm 1.599$ & $7.727 \pm 1.972$ & 0.016 \\
\hline Albumin (g/L) & $33.360 \pm 4.136$ & $29.997 \pm 3.911$ & $<0.001$ \\
\hline Globulin (g/L) & $25.175 \pm 3.618$ & $24.553 \pm 3.275$ & 0.128 \\
\hline WBC $\left(10^{9} / L\right)$ & $10.068 \pm 2.487$ & $10.698 \pm 3.174$ & 0.069 \\
\hline $\operatorname{RBC}\left(10^{9} / \mathrm{L}\right)$ & $3.743 \pm 0.411$ & $3.756 \pm 0.565$ & 0.796 \\
\hline $\mathrm{HB}(\mathrm{g} / \mathrm{L})$ & $105.620 \pm 14.201$ & $109.220 \pm 17.550$ & 0.062 \\
\hline $\operatorname{PLT}\left(10^{9} / \mathrm{L}\right)$ & $221.810 \pm 61.384$ & $218.670 \pm 69.487$ & 0.678 \\
\hline DBil $(\mu \mathrm{mol} / \mathrm{L})$ & $2.400(1.900-3.200)$ & $2.400(1.900-3.050)$ & 0.562 \\
\hline IBil $(\mu \mathrm{mol} / \mathrm{L})$ & $2.750(1.770-4.000)$ & $2.200(1.400-3.900)$ & 0.090 \\
\hline FFA (mmol/L) & $0.460(0.340-0.610)$ & $0.490(0.350-0.640)$ & 0.594 \\
\hline Glu (mmol/L) & $4.740 \pm 1.408$ & $5.050 \pm 1.292$ & 0.061 \\
\hline FH (yes/no) & $64.8 \% / 28.6 \%$ & $35.2 \% / 71.4 \%$ & 0.090 \\
\hline $\operatorname{EGFR}(\mathrm{ml} / \mathrm{min} / 1.73 \mathrm{~m} 2)$ & $222.933 \pm 66.143$ & $187.506 \pm 58.359$ & $<0.001$ \\
\hline
\end{tabular}

HCY Homocysteine, UA Uric acid, CYC Cysteine, TBA Total bile acid, BMI Body mass index, EGFR Estimated glomerular filtration rate, WBC White blood cell count, RBC Red blood cell count, HB Hemoglobin, PLT Platelets, DBil Direct bilirubin, IBil Indirect bilirubin, FFA Free fatty acids, Glu Glucose, FH Family history 
Table 2 Ordinary variables

\begin{tabular}{|c|c|c|c|c|c|}
\hline Item & 0 & 1 & 2 & 3 & 4 \\
\hline Age (yr) & $\leq 26.000$ & $(26.000,29.000]$ & $(29.000,31.000]$ & $(31.000,34.000]$ & $>34.000$ \\
\hline $\mathrm{HCY}(\mu \mathrm{mol} / \mathrm{L})$ & $\leq 7.930$ & $(7.930,12.000]$ & $(12.000,16.180]$ & $>16.180$ & \\
\hline UA ( $\mu \mathrm{mol} / \mathrm{L})$ & $\leq 236.000$ & $(236.000,294.000]$ & $(294.000,352.000]$ & $>352.000$ & \\
\hline CYC (mg/L) & $\leq 0.970$ & $(0.970,1.130]$ & $(1.130,1.350]$ & $>1.350$ & \\
\hline Albumin (g/L) & $\leq 29.830$ & $(29.830,32.600]$ & $(32.600,34.180]$ & $>34.180$ & \\
\hline TBA ( $\mu \mathrm{mol} / \mathrm{L})$ & $\leq 1.500$ & $(1.500,2.400]$ & $(2.400,4.000]$ & $>4.000$ & \\
\hline $\mathrm{Ca}(\mathrm{mmol} / \mathrm{L})$ & $\leq 2.060$ & $(2.060,2.140]$ & $(2.140,2.210]$ & $>2.210$ & \\
\hline Zinc ( $\mu \mathrm{mol} / \mathrm{L})$ & $\leq 7.000$ & $(7.000,8.000]$ & $(8.000,8.900]$ & $>8.900$ & \\
\hline BMI $\left(\mathrm{kg} / \mathrm{m}^{2}\right)$ & $\leq 26.350$ & $(26.350,29.320]$ & $(29.320,31.820]$ & $>31.820$ & \\
\hline EGFR (ml/min/1.73 m2) & $\leq 158.630$ & $(158.630,203.830]$ & $(203.830,254.130]$ & $>254.130$ & \\
\hline
\end{tabular}

CYC Cystatin C, UA Uric acid, HCY Homocysteine, EGFR Estimated glomerular filtration rate, BMI Body mass index, TBA Total bile acid, Ca Calcium

The above continuous variables with significant differences were converted into grade variables, shown in Table 2. According to the Chi-square, the $P$ values of age, HCY, CYC, UA, TBA, BMI, eGFR, ALB, calcium, zinc and family history of hypertensive disorders were less than 0.05 , showed statistically significant differences.

The 11 factors do not exist multicollinearity, the binary logistic model build by 11 variables is stable. The binary logistic regression showed ALB (0.489, 95\% CI: 0.335-0.714) was the protective factor. HCY $(1.400,95 \%$ CI: $1.051-$ 1.864), CYC (1.795, 95\% CI: 1.272-2.534), UA (1.667, 95\% CI: $1.205-2.306)$, BMI (1.589, 95\%CI: 1.161-2.175), age (1.289, 95\% CI: $1.026-1.620)$, and TBA $(1.721,95 \%$ CI: 1.277-2.320) were risk factors, showed on Table 3.

In order to optimize the model, logistic regression was performed again for 7 independent predictors, and the equation was: $\mathrm{Y}=(-5.855)+0.590 \quad(\mathrm{CYC})-0.766$ $(\mathrm{ALB})+0.591$ (UA) + 0.399 (BMI) +0.305 (age) +0.332 $(\mathrm{HCY})+0.526(\mathrm{TBA})$, as shown in Table 4. The AUC

Table 3 Binary logistic regression

\begin{tabular}{lllllll}
\hline Item & $\beta$ & $\mathrm{SE}$ & $\mathrm{P}$ & $\mathrm{OR}$ & $95 \% \mathrm{Cl}$ lower & $95 \% \mathrm{Cl}$ upper \\
\hline CYC & 0.585 & 0.176 & 0.001 & 1.795 & 1.272 & 2.534 \\
Albumin & -0.715 & 0.193 & $<0.001$ & 0.489 & 0.335 & 0.714 \\
UA & 0.511 & 0.166 & 0.002 & 1.667 & 1.205 & 2.306 \\
Calcium & -0.027 & 0.181 & 0.879 & 0.973 & 0.682 & 1.387 \\
Zinc & -0.107 & 0.149 & 0.471 & 0.898 & 0.671 & 1.203 \\
EGFR & -0.240 & 0.169 & 0.156 & 0.787 & 0.565 & 1.096 \\
BMI & 0.463 & 0.160 & 0.004 & 1.589 & 1.161 & 2.175 \\
Age & 0.254 & 0.116 & 0.029 & 1.289 & 1.026 & 1.620 \\
HCY & 0.336 & 0.146 & 0.021 & 1.400 & 1.051 & 1.864 \\
TBA & 0.543 & 0.152 & $<0.001$ & 1.721 & 1.277 & 2.320 \\
FH & 0.918 & 0.799 & 0.251 & 2.503 & 0.523 & 11.988 \\
Constant & -4.982 & 1.088 & 0.000 & 0.007 & & \\
\hline
\end{tabular}

CYC Cystatin C, UA Uric acid, HCY Homocysteine, EGFR Estimated glomerular filtration rate, $B M I$ Body mass index, TBA Total bile acid, FH Family history was $0.884(95 \% \mathrm{CI}: 0.848-0.921)$. The maximum value of the Youden index is 0.629 , the sensitivity is $88.0 \%$, and the specificity is $75.0 \%$, as shown in Fig. 1 . The scoring system was established according to the optimized model, and the scores of each factor were obtained, as shown in Table 5. ROC curve was drawn for the scoring system, with the AUC being 0.880 (95\%CI $0.842-0.918$ ). The maximum value of its Youden index is 0.606 , the sensitivity is $89.7 \%$, the specificity is $70.9 \%$, and the corresponding cut-off value is 15.5, as shown in Fig. 2.

\section{Discussion}

We collected 199 healthy pregnant women, 117 pregnant women with GH or PE and 22 variables. 11 indicators have significance and 7 indicators are independent risk factors. $\mathrm{HCY}$ is a special risk factor for hypertension to the Chinese Han population. Our results showed that $\mathrm{HCY}$ was closely associated with the incidence of newonset HDP in Chinese Han pregnant women [odds ratio (OR): $1.393,95 \% \mathrm{CI}: 1.054$ to 1.842 ] in the optimal model. HDP is an idiopathic disease with a complex mechanism that has a tremendous impact on the health of women and their children during pregnancy and the postpartum period. Studies are continuously being

Table 4 Optimal binary logistic regression

\begin{tabular}{lllccll}
\hline Item & $\beta$ & $\mathrm{SE}$ & $\mathrm{P}$ & $\mathrm{OR}$ & $95 \% \mathrm{Cl}$ lower & $95 \% \mathrm{Cl}$ upper \\
\hline CYC & 0.590 & 0.165 & $<0.001$ & 1.805 & 1.307 & 2.492 \\
Albumin & -0.766 & 0.155 & $<0.001$ & 0.465 & 0.343 & 0.630 \\
UA & 0.591 & 0.156 & $<0.001$ & 1.805 & 1.330 & 2.449 \\
BMI & 0.399 & 0.146 & 0.006 & 1.490 & 1.120 & 1.983 \\
Age & 0.305 & 0.109 & 0.005 & 1.356 & 1.096 & 1.678 \\
HCY & 0.332 & 0.142 & 0.020 & 1.393 & 1.054 & 1.842 \\
TBA & 0.526 & 0.147 & $<0.001$ & 1.692 & 1.269 & 2.256 \\
Constant & -5.855 & 0.964 & $<0.001$ & 0.003 & &
\end{tabular}

CYC Cystatin C, UA Uric acid, BMI Body mass index, HCY Homocysteine, TBA Total bile acid 


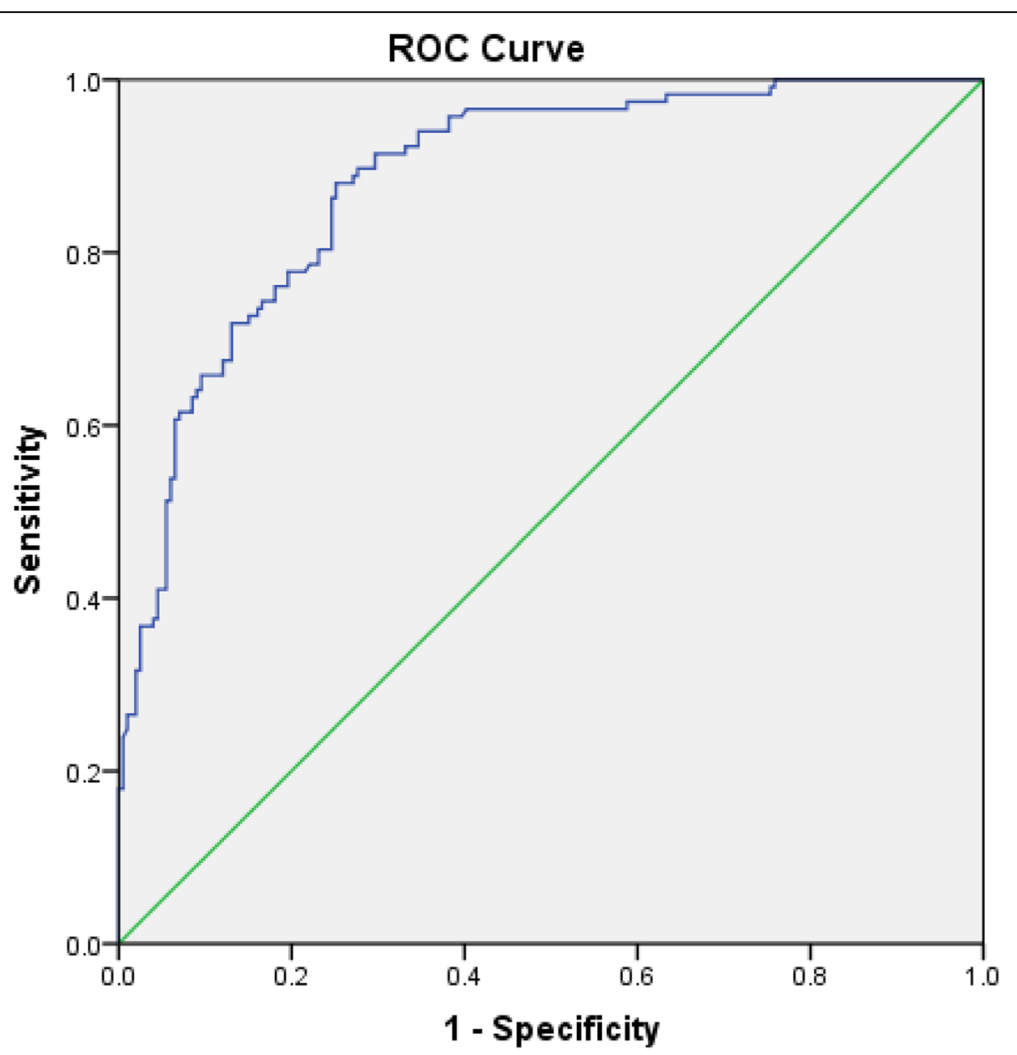

Fig. 1 ROC curve of binary logistic regression. The AUC was $0.884(95 \% \mathrm{Cl}: 0.848-0.921)$. The sensitivity is $88.0 \%$, and the specificity is $75.0 \%$

carried out on the risk factors that affect HDP development, and the guidelines are constantly changing. Therefore, risk factor-based models have been constantly designed and developed. Goetzinger and Verlohren et al. established a risk model for pregnant women based on high-risk factors and serum biochemical indicators such as pregnancy-associated plasma A (PAPP-A) and placenta growth factor (PIGF), but the receiver operating characteristic (ROC) curve was poor [16, 17]. The 2018 ISSHP guidelines classify HDP into 2 categories: chronic hypertension and new-onset hypertension ( $\mathrm{GH}$ or $\mathrm{PE}$ ) that occurred 20 weeks after pregnancy [3]. Therefore, in our study, GH and PE were classified as new-onset hypertension during pregnancy and were included in the case group, and normal pregnant women were used as the control group for the case-control study to establish a risk factor-based model for new-onset HDP in the Chinese Han population.

The multivariable analysis results in this study showed that albumin, CYC, UA, HCY, BMI, age, and TBA were independent predictive factors of HDP. Hyperhomocysteinemia is a specific disease in the Chinese Han population. The TT genotype caused by the $\mathrm{C}-\mathrm{T}$ mutation in the C677T gene polymorphism of the key metabolic enzyme MTHFR hinders the conversion of HCY to methionine and thus causes hyperhomocysteinemia. The population with the TT genotype accounts for $25 \%$ of the Chinese Han population, whereas the population with the TT genotype in North America and Europe accounts for only approximately $12 \%$ of the combined population [18]. Hyperhomocysteinemia causes vascular endothelial inflammation, which causes changes in vascular endothelial cells and the proliferation of smooth muscle cells, leading to vascular diseases [19]. Our study found that the risk of new-onset hypertension increases by nearly 1.4-fold with a quartile increase in HCY compared to control group. PE is a systemic endothelial dysfunction. HCY is likely related to the pathogenesis of HDP by vascular-mediated oxidative stress or direct injury [20]. Hyperhomocysteinemia is defined as a plasma HCY level greater than $10 \mu \mathrm{mol} / \mathrm{L}$ [21]. Our data showed that the rate of hyperhomocysteinemia in normal pregnant women was $55.8 \%$ and that the rate of hyperhomocysteinemia in patients with HDP was $76.1 \%$. This difference was statistically significant. Therefore, our study suggested that an increase in HCY increases the risk of HDP and that the population with HDP is more susceptible to hyperhomocysteinemia. The other study has demonstrated that the concentrations of $\mathrm{HCY}$ are associated with PE and the concentration of HCY in the first trimester was an independent risk factor for the development of severe PE [22]. A Large meta-analysis also 
Table 5 Scoring system

\begin{tabular}{|c|c|}
\hline Variance & Score \\
\hline \multicolumn{2}{|l|}{$\overline{\mathrm{CYC}}$} \\
\hline$\leq 0.970$ & 0 \\
\hline$(0.970,1.130]$ & 2 \\
\hline$(1.130,1.350]$ & 4 \\
\hline$>1.350$ & 6 \\
\hline \multicolumn{2}{|l|}{ Age } \\
\hline$\leq \mathbf{2 6 . 0 0 0}$ & 0 \\
\hline$(26.000,29 . .000]$ & 1 \\
\hline$(29.000,31.000]$ & 2 \\
\hline$(31.000,34.000]$ & 3 \\
\hline$>34.000$ & 4 \\
\hline \multicolumn{2}{|l|}{$\mathrm{HCY}$} \\
\hline$\leq 7.930$ & 0 \\
\hline$(7.930,12.000]$ & 1 \\
\hline$(12.000,16.180]$ & 2 \\
\hline$>16.180$ & 3 \\
\hline \multicolumn{2}{|l|}{ UA } \\
\hline$\leq 236.000$ & 0 \\
\hline$(236.000,294.000]$ & 2 \\
\hline$(294.000,352.000]$ & 4 \\
\hline$>352.000$ & 6 \\
\hline \multicolumn{2}{|l|}{ Albumin } \\
\hline$\leq \mathbf{2 9 . 8 3 0}$ & 0 \\
\hline$(29.830,32.600]$ & -2 \\
\hline$(32.600,34.180]$ & -4 \\
\hline$>34.180$ & -6 \\
\hline \multicolumn{2}{|l|}{ BMI } \\
\hline$\leq 26.350$ & 0 \\
\hline$(26.350,29.320]$ & 1 \\
\hline$(29.320,31.820]$ & 2 \\
\hline$>31.820$ & 3 \\
\hline \multicolumn{2}{|l|}{ TBA } \\
\hline$\leq 1.500$ & 0 \\
\hline$(1.500,2.400]$ & 2 \\
\hline$(2.400,4.000]$ & 4 \\
\hline$>4.000$ & 6 \\
\hline
\end{tabular}

CYCCystatin C, UA Uric acid, BMI Body mass index, HCY Homocysteine, TBA Total bile acid

indicated that the average HCY level was significantly higher in PE women than the normal pregnant women [23].

This study found that uric acid was also a risk factor for the development of HDP and that the risk of HDP increased by 1.805-fold for each quartile increase in uric acid. The finding is consistent with that of Nair et al. [24] and Wu et al. [25]. Some studies suggest that the increase in uric acid can reduce the production of nitric oxide in endothelial cells, leading to reduced trophoblast invasion and resulting in a lack of physiological transformation of the spiral artery, thus causing HDP, and that a deficiency in nitric oxide can lead to reduced vascular tension, resulting in PE [26]. Weissgerber and Brown believe that hyperuricemia leads to maternal endothelial dysfunction and affects placental blood flow, suggesting that uric acid is the precursor of $\mathrm{PE}[27,28]$. This is consistent with our findings. CYC is a relatively stable indicator that is not easily affected by nonrenal factors and can remain relatively stable throughout gestation [29]. Our study showed that CYC was positively correlated with the occurrence of HDP, which is consistent with the study by Novakov Mikic et al. [30].

Our study also showed that BMI was positively correlated with the occurrence of HDP and was an independent risk factor for the prediction of HDP, which was consistent with the studies by Marchi J [31] and Salihu [32]. Age is still a controversial clinical predictor of HDP. Some studies have shown that advanced age or young age is associated with a higher risk of HDP [33], while Paré et al. found that age greater than 40 years was significantly correlated with HDP [34]. There have also been many studies that suggest no significant correlation between age and the onset of HDP [35, 36]. Our study showed that age was positively correlated with the risk of HDP; the relative risk was 1.356 .

The pathophysiological basis of the development of HDP is vascular endothelial cell damage, which increases vascular permeability and can cause hypoalbuminemia and edema. Therefore, Dai et al. proposed that serum albumin is a protective factor for PE [37]. Our study showed that albumin was negatively correlated with the incidence of HDP; that is, the higher the serum albumin concentration was, the lower the risk of HDP. Albumin leakage is not limited to the interstitial space. Due to glomerular endothelial cell damage, the infiltration of albumin increases in PE. This symptom is not limited to $\mathrm{PE}$. The combined analysis of GH and PE showed that albumin was correlated with the risk of pregnancy induced hypertension; the risk was 0.489 . Even in the absence of proteinuria or edema in pregnant women with hypertension, decreased serum albumin concentration should be a cause of concern.

Total bile acid is an indicator that reflects intrahepatic cholestasis of pregnancy (ICP); ICP was once thought to be unrelated to liver diseases caused by PE; however, with progress in research, it was found that ICP may cause PE [38]. The diagnosis of ICP is complicated, and its evaluation indicators include pruritus and elevated liver enzymes. Total bile acid was the index that was focused on in this study, and it was found to be significantly positively correlated with new-onset hypertension. 


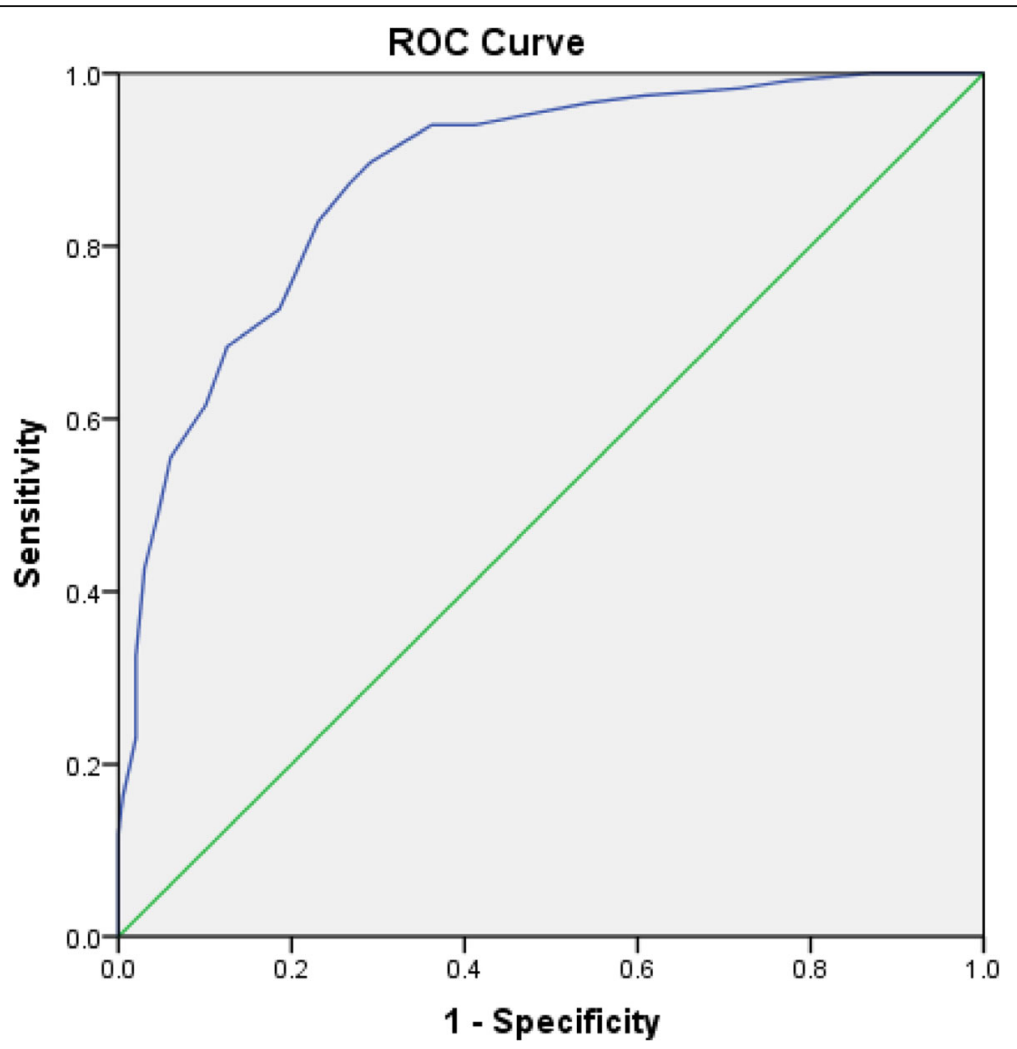

Fig. 2 ROC curve of scoring system. The AUC was $0.880(95 \% \mathrm{Cl} 0.842-0.918)$. The sensitivity is $89.7 \%$, the specificity is $70.9 \%$

The prevention and treatment of HDP relies on monitoring total bile acid.

The predictive model of risk factors for HDP provided in this study was used to analyze new-onset hypertension ( $\mathrm{GH}$ and $\mathrm{PE}$ ) as the case group. In the past 10 years, studies on HDP focused on PE risk prediction of but ignored GH. This was a case-control study comparing the risk factor-based models of HDP reported in case-control studies in the past 15 years. Direkvand-Moghadam et al. [39] established a prediction model using a medical history of PE, hypertension and infertility. The AUC of the model was 0.67 (95\% CI: $0.59-0.67, p<0.01$ ). The specific parameters, such as PIGF, free b-human chorionic gonadotropin ( $\beta$-hCG), PAPP-A and placental protein-13 (PP-13), which were not widely studied in clinical practice, were included in the study, and the model had a sensitivity of $70 \%$ [40]. Another study constructed the model that include the instructors reflective of placental function (PAPP-A and PlGF) and of the resistance in the uteroplacental circulation (uterine artery pulsatility index) could identify more than $90 \%$ of cases of early PE [41]. A large-scale systematic review summarized 29 risk prediction models for HDP, in which most of them predicted the risk of PE; the AUC of the prediction efficiency of the 29 models was between 0.67 and 0.90 [42]. Since then, most of the risk predictive model are included the maternal parameters, the instructors reflective of placental function such as PAPP-A, $\beta$-hCG or the PIGF. Little of the models covered the HCY or the kidney and liver function. The indicators included in our prediction model are more comprehensive, including maternal characteristics, family history, and metabolic indicators of the kidney and liver. We incorporated $\mathrm{HCY}$ and a series of biochemical indicators of liver and kidney function into our model innovatively. These indicators are easy to apply clinically and are cost-effective, and the AUC, sensitivity and specificity of our prediction model was 0.884 (95\% CI: 0.848 $0.921), 88.0 \%$, and $75.0 \%$, respectively. Compared to the aforementioned models, this model has high quality in terms of efficacy, and its sensitivity and specificity were excellent. As for other factors we have collected such as family history of HDP, in other study was proved to be the risk factor for HDP. Family history of HDP has been studied for a long time; a Norwegian cohort study now showed that pregnant women with a family history of HDP are more likely to develop hypertension during pregnancy, which is thought to be associated with family genetic phenotypes [43]. The genetic variants also have effect on 
HDP, among them; the M235T variant of the angiotensinogen gene has been shown in many subjects. In a Japanese study, the M235T frequency of the TT genotype is significantly higher in primigravid HDP (93-94\%) than controls (77\%) [44]. A meta-analysis suggested that MTHFR C677T polymorphism was associated with preeclampsia in Asian and Caucasian populations [14]. Because of genetic variants is not easy to carry out in clinical, we did not include it in the study. Other factors such as the intake of calcium have been proved to be the risk factors of HDP. According to a meta-analysis, the additional intake of calcium during pregnancy is an effective measure to reduce the incidence of preeclampsia, especially in populations at high risk [45]. Women who are primiparous also show a higher risk of HDP [2]. Some placenta-related factors may also predict the risk of HDP especially the PE. Increased production of soluble fms-like tyrosine kinase receptor-1 (sFlt-1) and soluble endoglin (sEng), and decreased circulating levels of free vascular endothelial growth factor (VEGF), contribute to the pathophysiology of PE. Cheng has studied the sFlt-1, sEng and VEGF levels showed a significant correlation with in the PE groups but not in the normotensive group [46]. Although the placenta-related factor has been studied widely in recent years, it is still difficult to carry out in clinical, so we did not include these factors in the study. The limitation of this prediction model is the lack of an external validation model. We are constantly collecting data to establish external validation models, and we will further improve the model according to the subsequent results to better serve in the clinical.

For clinical application, the risk factor-based prediction model for HDP in this study was $\mathrm{Y}=(-5.855)+$ 0.590 (CYC) -0.766 (ALB) + 0.591 (UA) + 0.399 (BMI) +0.305 (age) + $0.332(\mathrm{HCY})+0.526(\mathrm{TBA})$. The AUC of this model is 0.884 . For clinical, if we evaluated these factors together in the early gestational weeks, the predictive efficiency for later HDP was $88.4 \%$. We established a scoring system to simplify the risk evaluation. The AUC, sensitivity, specificity and cutoff value were $0.880,89.7 \%, 70.9 \%$, and 15.5 , respectively. The predictive efficiency of this scoring system is also more than $80 \%$. We tested the above 7 indicators for pregnant women, and the results were evaluated by the scoring system, those whose total score exceeded 15.5 were high-risk groups, while those whose total score was less than 15.5 were low-risk groups. In clinical, the 7 indicators are recommended for the screening of HDP, score can be made according to the indicators of pregnant women, and the risk stratification can be made according to the score. This predictive model has provided certain help to the judgment of doctors for early screening of high-risk groups and focused surveillance for this population.

\section{Conclusion}

Age, $\mathrm{HCY}, \mathrm{CYC}$, uric acid, total bile acid, BMI, and albumin were independent predictors of gestational hypertension and preeclampsia. The risk factor prediction model and scoring system established by these 7 items have high sensitivity and specificity, which can provide good estimates to the risk stratification of de novo hypertension in pregnancy.

\section{Abbreviations}

HDP: Hypertensive disorders of pregnancy; HELLP syndrome: Hemolysis, elevated liver enzymes, low platelet syndrome; ISSHP: International Society for the Study of hypertension in pregnancy; GH: Gestational hypertension; PE: Preeclampsia; PAPP-A: Pregnancy-associated plasma A ; PIGF: Placenta growth factor; ROC: Receiver operating characteristic; WBC: White blood cell count; RBC: Red blood cell count; HB: Haemoglobin; PLT: Platelets;

ALB: Albumin; GLB: Globulin; Glu: Glucose; FFA: Free fatty acids; DBil: Direct bilirubin; IBil: Indirect bilirubin; HCY: Homocysteine; CYC: Cystatin C; UA: Uric acid; TBA: Total bile acid; Ca: Serum calcium; Zn: Serum zinc; eGFR: Estimated glomerular filtration rate; AUC: Area under the receiver operating characteristic curve; Cl: Confidence interval; ICP: Intrahepatic cholestasis of pregnancy; $\beta$-hCG: $\beta$-human chorionic gonadotropin; PP-13: Placental protein-13; sFlt-1: Fms-like tyrosine kinase receptor-1; sEng: Soluble endoglin; VEGF: Vascular endothelial growth factor

\section{Acknowledgements}

None.

\section{Authors' contributions}

$\mathrm{YMH}$ and $\mathrm{LY}$ contributed equally to this work. YMH acquired, analyzed and interpreted data, wrote the manuscript. LY acquired, analyzed and interpreted data, revised the manuscript. RX designed the study, acquired, analyzed and interpreted the data. JRL revised the manuscript, LHZ acquired the data. All authors read and approved the final manuscript.

\section{Funding}

The laboratory test and analysis of indexes in pre-experiment was supported by the [Shandong Provincial Natural Science Foundation, China] under Grant [No.

ZR2014HP046]; the enrollment of patients and the laboratory test of biomarkers were supported by the [Shandong Provincial Science and Technology Development Programme Foundation, China] under Grant [No. 2017WS462] and [Shandong Provincial Key Research and Development Programme Foundation, China] under Grant [No. 2018GSF118009]; the data analysis and the polish fee were supported by the [Technology Programme Foundation of Jinan, China] under Grant [No. 201821007].

\section{Availability of data and materials}

Study protocol and data set: Not available. Statistical code: Available from Dr. Xu (e-mail, xuruicn@hotmail.com).

\section{Ethics approval and consent to participate}

The ethics committee of Jinan maternity and child care hospital approved the study, and all patients gave written informed consent for participating in the study.

\section{Consent for publication}

Not applicable.

\section{Competing interests}

No benefits in any form have been received or will be received from a commercial party related directly or indirectly to the subject of this article.

\section{Author details}

${ }^{1}$ Department of Cardiology, Shandong Provincial Qianfoshan Hospital, Shandong University, Jinan 250014, P.R. China. ${ }^{2}$ Department of Cardiology, 
The First Affiliated Hospital of Shandong First Medical University, Jinan 250014, P.R. China. ${ }^{3}$ Department of Medicine, Jinan Maternity and Child Care Hospital, Jinan 250001, P.R. China. ${ }^{4}$ Department of Cardiology, Shandong Provincial Third Hospital, Jinan 250031, P.R. China.

\section{Received: 20 November 2019 Accepted: 12 March 2020 Published online: 03 April 2020}

\section{References}

1. Kobashi G. Genetic and environmental factors associated with the development of hypertension in pregnancy. J Epidemiol. 2006;16(1):1-8.

2. Ye C, Ruan Y, Zou L, Li G, Li C, Chen Y, et al. The 2011 survey on hypertensive disorders of pregnancy (HDP) in China: prevalence, risk factors, complications, pregnancy and perinatal outcomes. PLoS One. 2014;9(6): e100180.

3. Brown MA, Magee LA, Kenny LC, Karumanchi SA, McCarthy FP, Saito S, et al. Hypertensive disorders of pregnancy: ISSHP classification, diagnosis, and management recommendations for international practice. Hypertension. 2018;72(1):24-43.

4. Umesawa M, Kobashi G. Epidemiology of hypertensive disorders in pregnancy: prevalence, risk factors, predictors and prognosis. Hypertens Res. 2017:40(3):213-20

5. Morikawa M, Yamada T, Yamada T, Cho K, Sato S, Minakami H. Seasonal variation in the prevalence of pregnancy-induced hypertension in Japanese women. J Obstet Gynaecol Res. 2014;40(4):926-31.

6. Algert CS, Roberts CL, Shand AW, Morris JM, Ford JB. Seasonal variation in pregnancy hypertension is correlated with sunlight intensity. Am J Obstet Gynecol. 2010;203(3):215 e1-5.

7. Ray JG, Vermeulen MJ, Schull MJ, Redelmeier DA. Cardiovascular health after maternal placental syndromes (CHAMPS): population-based retrospective cohort study. Lancet. 2005;366(9499):1797-803.

8. Wu P, Haththotuwa R, Kwok CS, Babu A, Kotronias RA, Rushton C, et al. Preeclampsia and Future Cardiovascular Health: A Systematic Review and Meta-Analysis. Circ Cardiovasc Qual Outcomes. 2017;10(2):e003497.

9. Best LG, Lunday L, Webster E, Falcon GR, Beal JR. Pre-eclampsia and risk of subsequent hypertension: in an American Indian population. Hypertens Pregnancy. 2017:36(2):131-7.

10. Alsnes IV, Vatten LJ, Fraser A, Bjorngaard JH, Rich-Edwards J, Romundstad PR, et al. Hypertension in pregnancy and offspring cardiovascular risk in young adulthood: prospective and sibling studies in the HUNT study (NordTrondelag health study) in Norway. Hypertension. 2017;69(4):591-8.

11. Pare E, Parry S, McElrath TF, Pucci D, Newton A, Lim KH. Clinical risk factors for preeclampsia in the 21st century. Obstet Gynecol. 2014;124(4):763-70.

12. Yong HE, Melton PE, Johnson MP, Freed KA, Kalionis B, Murthi P, et al. Genome-wide transcriptome directed pathway analysis of maternal preeclampsia susceptibility genes. PLoS One. 2015;10(5):e0128230.

13. Shinya K, Nakayama T, Nakayama T, Yamamoto T. A case-control study between the STIM1 gene and hypertensive disorders of pregnancy. Hypertens Res. 2018;41(1):39-44.

14. Yang B, Fan S, Zhi X, Li Y, Liu Y, Wang D, et al. Associations of MTHFR gene polymorphisms with hypertension and hypertension in pregnancy: a metaanalysis from 114 studies with 15411 cases and 21970 controls. PLoS One. 2014;9(2):e87497.

15. Rodriguez-Lopez M, Wagner P, Perez-Vicente R, Crispi F, Merlo J. Revisiting the discriminatory accuracy of traditional risk factors in preeclampsia screening. PLoS One. 2017;12(5):e0178528.

16. Goetzinger KR, Tuuli MG, Cahill AG, Macones GA, Odibo AO. Development and validation of a risk factor scoring system for first-trimester prediction of preeclampsia. Am J Perinatol. 2014:31(12):1049-56.

17. Verlohren S, Perschel FH, Thilaganathan B, Droge LA, Henrich W, Busjahn A, et al. Angiogenic markers and cardiovascular indices in the prediction of hypertensive disorders of pregnancy. Hypertension. 2017;69(6):1192-7.

18. Zhao M, Wang X, He M, Qin X, Tang G, Huo Y, et al. Homocysteine and stroke risk: modifying effect of Methylenetetrahydrofolate Reductase C677T polymorphism and folic acid intervention. Stroke. 2017:48(5):1183-90.

19. Antoniades C, Antonopoulos AS, Tousoulis D, Marinou K, Stefanadis C. Homocysteine and coronary atherosclerosis: from folate fortification to the recent clinical trials. Eur Heart J. 2009:30(1):6-15.

20. Cheng PJ, Huang SY, Su SY, Hsiao CH, Peng HH, Duan T. Prognostic value of cardiovascular disease risk factors measured in the first-trimester on the severity of preeclampsia. Medicine (Baltimore). 2016;95(5):e2653.
21. Sacco RL, Adams R, Albers G, Alberts MJ, Benavente O, Furie K, et al. Guidelines for prevention of stroke in patients with ischemic stroke or transient ischemic attack: a statement for healthcare professionals from the American Heart Association/American Stroke Association Council on stroke: co-sponsored by the council on cardiovascular radiology and intervention: the American Academy of Neurology affirms the value of this guideline. Stroke. 2006;37(2):577-617.

22. Sun F, Qian W, Zhang C, Fan J-X, Huang H-F. Correlation of maternal serum Homocysteine in the first trimester with the development of gestational hypertension and preeclampsia. Med Sci Monit. 2017;23:5396-401.

23. Gaiday AN, Tussupkaliyev AB, Bermagambetova SK, Zhumagulova SS, Sarsembayeva LK, Dossimbetova MB, et al. Effect of homocysteine on pregnancy: a systematic review. Chem Biol Interact. 2018;293:70-6.

24. Nair A, Savitha C. Estimation of serum uric acid as an Indicator of severity of preeclampsia and perinatal outcome. J Obstet Gynaecol India. 2017:67(2): 109-18.

25. Wu $Y$, Xiong $X$, Fraser WD, Luo ZC. Association of uric acid with progression to preeclampsia and development of adverse conditions in gestational hypertensive pregnancies. Am J Hypertens. 2012;25(6):711-7.

26. Bainbridge SA, Roberts JM. Uric acid as a pathogenic factor in preeclampsia. Placenta. 2008:29(Suppl A):S67-72.

27. Martin AC, Brown MA. Could uric acid have a pathogenic role in preeclampsia? Nat Rev Nephrol. 2010;6(12):744-8.

28. Weissgerber TL, Milic NM, Turner ST, Asad RA, Mosley TH Jr, Kardia SL, et al. Uric acid: a missing link between hypertensive pregnancy disorders and future cardiovascular disease? Mayo Clin Proc. 2015:90(9):1207-16.

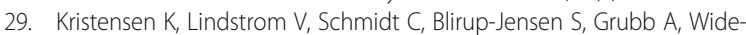
Swensson D, et al. Temporal changes of the plasma levels of cystatin C, beta-trace protein, beta2-microglobulin, urate and creatinine during pregnancy indicate continuous alterations in the renal filtration process. Scand J Clin Lab Invest. 2007;67(6):612-8.

30. Novakov Mikic A, Cabarkapa V, Nikolic A, Maric D, Brkic S, Mitic G, et al. Cystatin C in pre-eclampsia. J Matern Fetal Neonatal Med. 2012;25(7):961-5.

31. Marchi J, Berg M, Dencker A, Olander EK, Begley C. Risks associated with obesity in pregnancy, for the mother and baby: a systematic review of reviews. Obes Rev. 2015:16(8):621-38.

32. Sailu. HM. Does maternal obesity cause preeclampsia A systematic review of the evidence. Minerva Ginecol. 2012:64:259-80.

33. Abalos E, Cuesta C, Carroli G, Qureshi Z, Widmer M, Vogel JP, et al. Preeclampsia, eclampsia and adverse maternal and perinatal outcomes: a secondary analysis of the World Health Organization multicountry survey on maternal and newborn health. BJOG. 2014;121(Suppl 1):14-24.

34. Bartsch E, Medcalf KE, Park AL, Ray JG. High risk of pre-eclampsia identification G. Clinical risk factors for pre-eclampsia determined in early pregnancy: systematic review and meta-analysis of large cohort studies. BMJ. 2016;353:11753

35. Conde-Agudelo A, Belizan JM. Risk factors for pre-eclampsia in a large cohort of Latin American and Caribbean women. BJOG. 2000;107(1):75-83.

36. Coonrod DV, Hickok DE, Zhu K, Easterling TR, Daling JR. Risk factors for preeclampsia in twin pregnancies: a population-based cohort study. Obstet Gynecol. 1995;85(5 Pt 1):645-50.

37. Dai DM, Cao J, Yang HM, Sun HM, Su Y, Chen YY, et al. Hematocrit and plasma albumin levels difference may be a potential biomarker to discriminate preeclampsia and eclampsia in patients with hypertensive disorders of pregnancy. Clin Chim Acta. 2017:464:218-22.

38. Wikstrom Shemer E, Marschall HU, Ludvigsson JF, Stephansson O. Intrahepatic cholestasis of pregnancy and associated adverse pregnancy and fetal outcomes: a 12-year population-based cohort study. BJOG. 2013; 120(6):717-23.

39. Direkvand-Moghadam A, Khosravi A, Sayehmiri K. Predictive factors for preeclampsia in pregnant women: a receiver operation character approach. Arch Med Sci. 2013;9(4):684-9.

40. Di Lorenzo G, Ceccarello M, Cecotti V, Ronfani L, Monasta L, Vecchi Brumatti $L$, et al. First trimester maternal serum PIGF, free beta-hCG, PAPP-A, PP-13, uterine artery Doppler and maternal history for the prediction of preeclampsia. Placenta. 2012;33(6):495-501.

41. Poon LC, Kametas NA, Maiz N, Akolekar R, Nicolaides KH. First-trimester prediction of hypertensive disorders in pregnancy. Hypertension. 2009;53(5): 812-8.

42. Al-Rubaie Z, Askie LM, Ray JG, Hudson HM, Lord SJ. The performance of risk prediction models for pre-eclampsia using routinely collected maternal 
characteristics and comparison with models that include specialised tests and with clinical guideline decision rules: a systematic review. BJOG. 2016; 123(9):1441-52.

43. Roten LT, Thomsen LC, Gundersen AS, Fenstad MH, Odland ML, Strand KM, et al. The Norwegian preeclampsia family cohort study: a new resource for investigating genetic aspects and heritability of preeclampsia and related phenotypes. BMC Pregnancy Childbirth. 2015;15:319.

44. Kobashi G, Hata A, Shido K. Association of a Variant of the angiotensinogen gene with pure type of hypertension in pregnancy in the Japanese: implication of a racial difference and significance of an age factor. Am J Med Genet. 1999:86:232-6.

45. Patrelli TS, Dall'asta A, Gizzo S, Pedrazzi G, Piantelli G, Jasonni VM, et al. Calcium supplementation and prevention of preeclampsia: a meta-analysis. J Matern Fetal Neonatal Med. 2012;25(12):2570-4.

46. Cheng M, He P, Fu J. The relationship between circulating tissue transglutaminase, soluble fms-like tyrosine kinase-1, soluble endoglin and vascular endothelial growth factor in pre-eclampsia. J Hum Hypertens. 2016; 30(12):788-93.

\section{Publisher's Note}

Springer Nature remains neutral with regard to jurisdictional claims in published maps and institutional affiliations.

- fast, convenient online submission

- thorough peer review by experienced researchers in your field

- rapid publication on acceptance

- support for research data, including large and complex data types

- gold Open Access which fosters wider collaboration and increased citations

- maximum visibility for your research: over $100 \mathrm{M}$ website views per year

At BMC, research is always in progress. 